\title{
Quality of life outcomes in patients with chronic myeloid leukemia treated with tyrosine kinase inhibitors: a controlled comparison
}

\author{
Kristin M. Phillips • Javier Pinilla-Ibarz • \\ Eduardo Sotomayor • Morgan R. Lee • \\ Heather S. L. Jim • Brent J. Small • Lubomir Sokol • \\ Jeffrey Lancet • Sara Tinsley • Kendra Sweet • \\ Rami Komrokji • Paul B. Jacobsen
}

Received: 3 July 2012 / Accepted: 14 October 2012 / Published online: 20 November 2012

(C) Springer-Verlag Berlin Heidelberg 2012

\begin{abstract}
Purpose Tyrosine kinase inhibitors (TKIs) are now standard treatment for chronic myeloid leukemia (CML). While TKIs have less toxicity than previous treatments, they have side effects that can impact quality of life (QOL).

Methods This study compared CML patients taking a TKI for an average of 4.01 years (range $0.50-9.79$ years) to ageand gender-matched controls with no history of cancer on measures of symptom burden, depression, fatigue, sleep, and health-related QOL.

Results Compared to controls $(n=62)$, CML patients $(n=$ 62) taking a TKI (imatinib $55 \%$, nilotinib $31 \%$, and dasatinib $14 \%$ ) reported significantly worse fatigue severity $(p<.001)$, fatigue interference $(p<.001)$, depression
\end{abstract}

K. M. Phillips

Mental Health and Behavioral Sciences,

James A. Haley Veterans' Hospital,

Tampa, FL, USA

J. Pinilla-Ibarz $\cdot$ E. Sotomayor $\cdot$ L. Sokol $\cdot$ J. Lancet $\cdot$ S. Tinsley $\cdot$

K. Sweet $\cdot$ R. Komrokji

Department of Malignant Hematology, Moffitt Cancer Center,

Tampa, FL, USA

M. R. Lee

Department of Psychology, University of South Florida,

Tampa, FL, USA

H. S. L. Jim • B. J. Small • P. B. Jacobsen $(\bowtie)$

Department of Health Outcomes and Behavior,

Moffitt Cancer Center,

Tampa, FL, USA

e-mail: Paul.Jacobsen@moffitt.org

B. J. Small

School of Aging Studies, University of South Florida,

Tampa, FL, USA $(p=.007)$, symptom burden $(p<.001)$, and physical QOL $(p<.001)$. TKI patients were also more likely meet established cutoffs for clinically meaningful fatigue ( $p$ values $<.001)$ and depression $(p=.004)$. There were no differences in mental QOL or sleep ( $p$ values>.010). Regarding specific symptoms, TKI patients were more likely to report nausea, diarrhea, itching, skin changes, swelling of arms or legs, and not looking like themselves ( $p$ values $<.001$ ).

Conclusions These data suggest the need for interventions to address QOL in CML patients taking TKIs.

Keywords Cancer $\cdot$ Quality of life $\cdot$ Depression $\cdot$ Fatigue

\section{Introduction}

Over the past decade, tyrosine kinase inhibitors (TKIs) have become the standard treatment for chronic myeloid leukemia (CML). The majority of the research on quality of life (QOL) among patients taking TKIs for CML has focused on imatinib, the first TKI approved as a treatment for CML [1]. One of the initial studies on this topic was a randomized trial that compared patients treated with imatinib to patients treated with interferon alfa (INF $\alpha$ ) plus low-dose cytarabine [2]. Compared to those taking IFN $\alpha$ and cytarabine, patients taking imatinib reported significantly better QOL [2]. A more recent prospective, single-arm study reported that QOL improved over time among CML patients taking imatinib [3].

These studies suggest that TKIs prescribed to CML patients have less deleterious effects on QOL than earlier forms of treatment [2] and can produce improvements in QOL over pretreatment baselines [3]. A key issue not addressed by these studies is how QOL in CML patients 
taking TKIs compares to that of similar individuals in the general population. Because TKIs have side effects [1] and intolerance is a problem for some CML patients $[4,5]$, is it likely that TKIs can have an adverse affect on QOL. To address this issue, Efficace et al. [6] used the Medical Outcomes Study 36-Item Short Form Health Survey (SF-36) to compare QOL among CML patients on imatinib for a median of 5 years (range 3.0-9.3 years) with published population norms [7], adjusted for relevant demographic variables (e.g., age and sex). Results indicated patients had significantly worse overall physical, but not mental QOL, relative to adjusted population norms. Additional analyses indicated differences in QOL between patients and population norms were particularly pronounced among females and younger individuals (ages 18-39 years).

The current study sought to extend prior research by Efficace et al. [6] by examining a broader range of QOL outcomes. Toward this end, participants in this study completed measures of fatigue, depression, symptom burden, and sleep as well as the SF-36 measure of health-related QOL. The current study also sought to provide a direct comparison of patients' QOL with that of similar individuals. Accordingly, rather than relying on general population norms, we recruited a sample of individuals with no history of cancer matched on age and gender to a sample of CML patients taking TKIs. The primary aim was to compare QOL outcomes (i.e., fatigue, depression, sleep, symptom burden, and health-related QOL) in the two groups. We hypothesized that patients taking a TKI would report worse fatigue, depressive symptoms, symptom burden, sleep, and healthrelated QOL relative to the comparison group. A second aim was to identify differences in symptom distress between the two groups. Based on reports of common side effects of TKIs [1], we hypothesized that patients taking a TKI would report greater distress related to fluid retention, gastrointestinal upset, pain, and skin changes.

\section{Methods}

Participants and procedures

Potential patient participants were identified using a registry maintained by the Moffitt Cancer Center (MCC) Hematologic Malignancies Program. Eligibility criteria were: 18 years or older; able to speak and read English; diagnosed with CML in the chronic phase; receiving treatment for CML at MCC; had achieved a complete cytogenic response; were being treated with imatinib, nilotinib, or dasatinib; had been treated with a TKI for a minimum of 6 months; had no change in TKI dose in the past 2 months; and had a mailing address and a working telephone number. Eligible patients with scheduled appointments at MCC in the next 3 months were recruited in person; those without scheduled appointments in the next 3 months were recruited via mail. For inperson recruitment, patients who provided written informed consent were given a questionnaire they could complete during their visit or take home and return in a postage-paid envelope. For by-mail recruitment, potential participants were sent a package containing two copies of an informed consent (one to sign and return and one for participants to keep), a questionnaire, a postage-paid envelope, a decline card, and a letter describing the study. The letter requested that patients call a toll-free telephone number or return the decline card within a week if they did not want to be contacted about the study. Individuals who did not call the toll-free number or return the decline card within 10 days of the package being sent were called by a member of the study team to confirm eligibility and determine whether they wished to participate. Up to five attempts at different times on separate days were made to contact each individual. For individuals who verbally agreed to participate but did not return their informed consents or questionnaires within 3 weeks, up to four attempts were made to reach them via telephone.

Potential noncancer control participants were individuals living in the same geographic region as the patients. They were identified, as in our prior research [8], using information obtained from Marketing Systems Group (MSG), Inc. (Fort Washington, PA, USA). Eligibility criteria for nonpatients were: 18 years or older, able to speak and read English, same gender as the patient to whom they were being matched, within 5 years of the age of the patient to whom they were being matched, no self-reported history of cancer (other than nonmelanoma skin cancer), and had a mailing address and a working telephone number. Noncancer control participants were recruited using mail and telephone procedures described previously for patients. A list of up to 25 potential noncancer matches was prepared for each patient. Potential noncancer controls were randomly selected from the list until a matched noncancer control participant had been successfully recruited and returned a completed questionnaire.

Each participant was compensated $\$ 20$ for completing the study questionnaire. The study was approved by the University of South Florida Institutional Review Board and all participants provided written informed consent.

\section{Measures}

Demographic characteristics were assessed via self-report. Clinical characteristics of TKI patients were obtained through medical record review. Fatigue was assessed using the fatigue interference and fatigue severity subscales of the Fatigue Symptom Inventory (FSI) [9]. Fatigue interference was obtained by averaging seven items assessing how much 
fatigue had interfered in the past week with: general level of activity, ability to bathe and dress, normal work activity, ability to concentrate, relations with other people, enjoyment of life, and mood $(0=$ no interference, $10=$ extreme interference). The fatigue severity score was obtained by averaging four items assessing most, least, and average fatigue in the past week and current fatigue $(0=$ not at all fatigued, $10=$ as fatigued as I could be). Prior work demonstrated the FSI to be a reliable and valid measure of fatigue in cancer patients $[9,10]$. A score of 3 or higher has been established as a cutoff for clinically meaningful fatigue [11].

Depression was assessed using the Center for Epidemiological Studies Depression (CES-D) scale [12]. The 20-item CES-D assesses how often participants have experienced depressive symptoms in the past week $(0=$ none of the time, $3=$ most of the time). Total scores can range from 0 to 60 . Prior research has demonstrated the validity of the CES-D with cancer patients [13]. A score of 16 or higher has been established as a cutoff for clinically significant depression [12].

Sleep was assessed using the global score of the Pittsburg Quality Sleep Index (PSQI) [14]. The global score is the sum of seven components (sleep quality, sleep latency, sleep duration, sleep efficiency, sleep disturbance, use of sleep medication, and daytime dysfunction). Each component is rated on a 0 to 3 scale, with higher score indicating worse sleep. The PSQI has been shown to be a valid measure of sleep in cancer patients $[15,16]$. A score greater than 5 has been established as a cutoff for clinically significant sleep disturbances [14].

Symptom burden was assessed with Part 1 of the Memorial Symptom Assessment Scale-Short Form (MSAS-SF) [17]. The original version consists of 28 items. However, for the purposes of these analyses, two items were removed (i.e., "lack of energy" and "difficulty sleeping") to reduce overlap with measures of fatigue and sleep. Participants were asked whether they experienced each symptom (yes/ no). Symptoms that were not endorsed received a score of 0 . Symptoms that were endorsed were scored based participants' ratings of how much it distressed or bothered them: 'not at all' (0.8), 'a little bit' (1.6), 'somewhat' (2.4), 'quite a bit' (3.2), and 'very much' (4.0) [17]. Ratings for the 26 items were averaged, with higher scores indicating more symptom burden.

Health-related quality of life was assessed using the Medical Outcomes Survey 36-item Short Form (SF-36) $[18,19]$. The acute version used in this study asks about perceived health and functioning over the past week. The SF-36 contains eight scales: physical functioning (PF), role physical (RP), bodily pain (BP), general health (GH), vitality (VT), social functioning (SF), role emotional (RE), and mental health (MH). The SF-36 also yields two summary scores: physical component summary (PCS) and mental component summary (MCS). Higher scores indicate better QOL. The SF-36 has been shown to be a valid and reliable measure with cancer patients [19].

\section{Statistical analysis}

Analyses were conducting using SAS version 9.1.3 (SAS Institute, Cary, NC, USA). Prior to the main analysis, $t$ tests and chi-square tests were used to compare the two groups on continuous and categorical demographic variables, respectively. The primary outcome was the effect of group (TKI vs. NC) on fatigue, depression, symptom burden, and health-related QOL. A secondary analysis compared the two groups on the specific symptoms reported. To achieve these aims, we performed analyses of variance (ANOVAs). Due to the large number of comparisons, results were considered significant at $p<.01$. Effect sizes were calculated using Cohen's $d$, with an effect size of 0.2 to 0.3 considered small, 0.5 medium, and 0.8 or above large [20]. Analyses were also conducted controlling for demographic variables for which there were differences between groups at $p<.10$. In addition, chi-square tests were conducted to determine whether TKI participants were more likely to meet clinical cutoffs for fatigue, depression, and sleep. Group differences in clinical cutoffs were considered significant at $p<.01$.

\section{Results}

\section{Participant accrual}

Of 367 patients screened, 99 met eligibility criteria and 71 provided informed consent. Of the 71 patients who provided informed consent, four did not complete the questionnaire and five became ineligible after consent, resulting in 62 patient participants. Of the 385 nonpatients mailed recruitment letters, 14 were never contacted due to incorrect mailing addresses, nine were ineligible, and 76 provided informed consent and returned the questionnaire. Because we used one-to-one matching, data for the 62 nonpatients closest in age to the patients were retained for analysis.

\section{Demographic characteristics}

Participants $(N=124 ; 52 \%$ male; mean $=55$ years, range 18 81 years) were CML patients receiving a TKI (TKI, $n=62$ ) and people without cancer (NC, $n=62)$ matched on age and gender. There were no group differences on age, gender, education, or marital status. However, patients were less likely to be Caucasian $(p=.023)$ and there was a trend for patients to be more likely to be Hispanic ( $p=.062$; see Table 1). Accordingly, race and ethnicity were included as covariates in analyses described below. 
Table 1 Demographic characteristics of study participants. $N=124$

\begin{tabular}{llll}
\hline Variable & TKI group $n=62$ & NC group n=62 & $\mathrm{p}^{\mathrm{a}}$ \\
\hline Age at study entry (years) & $55.43(14.54)$ & $56.22(14.69)$ & .764 \\
Education (\%) & & & .590 \\
$\quad$ College graduates & 45 & 50 & \\
$\quad$ Noncollege graduates & 55 & 50 & 1.00 \\
Gender (\%) & & & \\
$\quad$ Females & 48 & 48 & .023 \\
$\quad$ Males & 52 & 52 & \\
Race (\%) & & & .062 \\
$\quad$ White & 82 & 95 & \\
$\quad$ Non-White & 18 & 5 & .350 \\
Ethnicity (\%) & & & \\
$\quad$ Hispanic & 11 & 2 & \\
$\quad$ Non-Hispanic & 89 & 98 & \\
Marital status (\%) & & 32 & \\
$\quad$ Married & 60 & 40 &
\end{tabular}

TKI group patients treated with tyrosine kinase inhibitors, NC group noncancer participants

${ }^{a} p$ values are based on chi-square analyses for categorical variables and $t$ tests for continuous variables

\section{Clinical characteristics}

Patients had been diagnosed with CML for an average of 4.55 years $(\mathrm{SD}=2.80$, range $0.57-12.79$ years) and were taking imatinib $(n=34,55 \%)$, nilotinib $(n=19,31 \%)$, or dasatinib $(n=9,14 \%)$. Approximately half the participants ( $n=33,53 \%$ ) were on their first TKI treatment. Patients had been on their current TKI for an average of 3.02 years $(\mathrm{SD}=$ 2.12, range $0.04-8.34$ years) and on any TKI for 4.01 years $(\mathrm{SD}=2.42$, range $0.50-9.79$ years). The majority of patients were in the low Sokal risk group $(n=45,73 \%)$. All patients had achieved current hematologic response, current cytogenic response, and current major molecular response. Current complete molecular response (i.e., "undetectable" BCR-ABL) was evident in 27 patients (44\%; see Table 2).

\section{Continuous QOL outcomes}

As shown in Table 3, there were significant group differences $(p$ values $\leq .009)$ and medium to large effect sizes for fatigue interference (FSI interference), fatigue severity (FSI severity), depression (CES-D), symptom burden (MSASSF), SF-36 physical component score (PCS), and the following SF-36 subscales: physical functioning (PF), role physical (RP), general health $(\mathrm{GH})$, vitality (VT), social functioning (SF), and role emotional (RE). For each significant finding, TKI participants reported worse functioning than NC participants (see Table 3). There were no group
Table 2 Clinical characteristics of patient participants. $N=62$

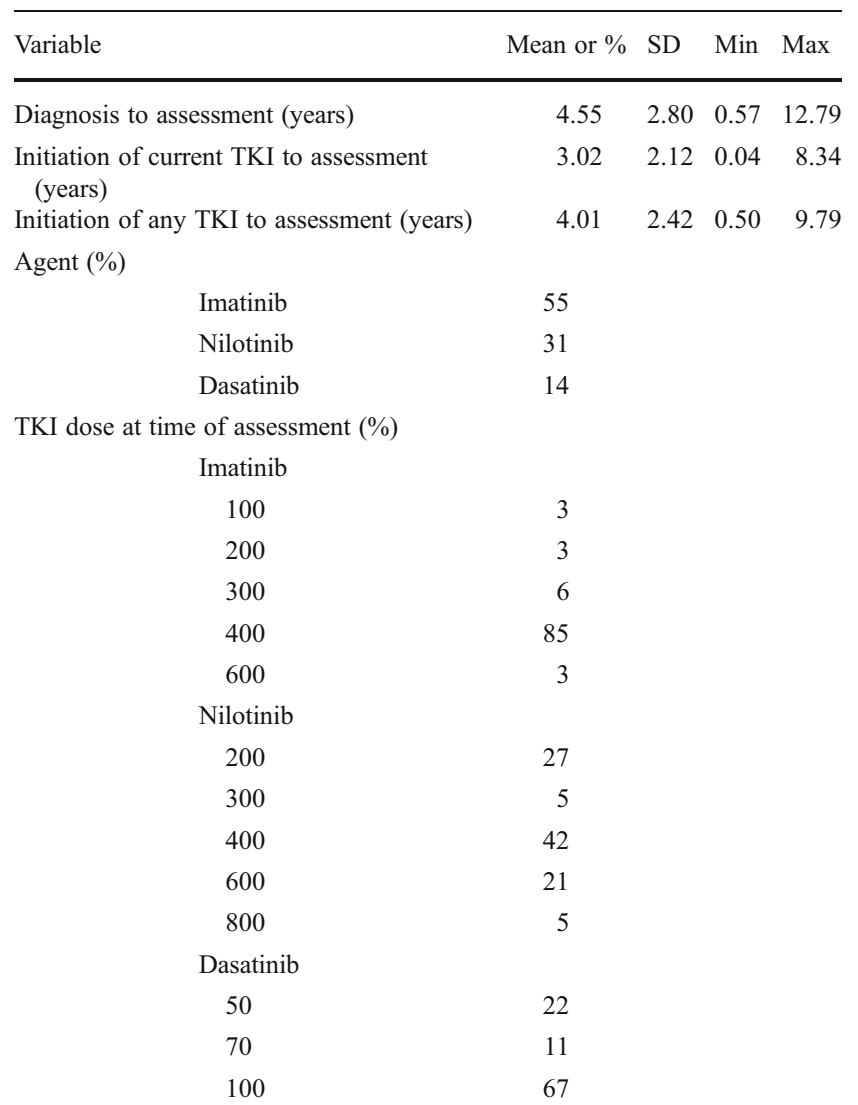

Starting dose of current TKI (\%)

$\begin{array}{cr}\text { Imatinib } & \\ 400 & 88 \\ 600 & 3 \\ 800 & 9 \\ \text { Nilotinib } & \\ 200 & 21 \\ 300 & 5 \\ 400 & 58 \\ 600 & 11 \\ 800 & 5 \\ \text { Dasatinib } & \end{array}$

$\begin{array}{ll}50 & 11 \\ 70 & 45 \\ 100 & 44\end{array}$

Sokal risk group (\%)

$\begin{array}{ll}\text { Low } & 73 \\ \text { Intermediate } & 16 \\ \text { High } & 11\end{array}$

Previous TKI treatments (\%)

0

53
1
40

227

Current hematologic0020response (\%) 100

Current cytogenic response (\%) 100

Current major molecular response (\%) 100

Current complete molecular response (\%) 44 
Table 3 Unadjusted means and standard deviations on quality of life outcomes. $N=124$

For SF-36 scores, higher scores indicate better functioning; for all other variables, higher scores indicate worse functioning

TKI patients treated with tyrosine kinase inhibitors, $N C$ noncancer participants, $Q O L$ quality of life, FSI Fatigue Severity Index, $C E S-D$ Center for Epidemiological Studies Depression, MSAS-SF Memorial Symptom Assessment Scale-Short Form, PSQI Pittsburg Quality Sleep Index, SF-36 Medical Outcomes Survey 36-item Short Form

\begin{tabular}{lccrc}
\hline Measure & TKI, mean (SD) & NC, mean (SD) & $\mathrm{p}$ & Cohen's $d$ \\
\hline Fatigue interference (FSI interference) & $2.99(2.82)$ & $1.07(1.36)$ & $<.001$ & 0.87 \\
Fatigue severity (FSI severity) & $3.99(2.26)$ & $2.31(1.47)$ & $<.001$ & 0.88 \\
Depression (CES-D total) & $13.56(10.67)$ & $8.74(8.65)$ & .007 & 0.50 \\
Sleep (PSQI global) & $6.18(4.20)$ & $4.89(3.53)$ & .065 & 0.33 \\
Symptom burden (MSAS-SF) & $0.71(0.64)$ & $0.29(0.24)$ & $<.001$ & 0.87 \\
Physical QOL (SF-36 PCS) & $40.44(12.36)$ & $48.93(8.48)$ & $<.001$ & -0.80 \\
Mental QOL (SF-36 MCS) & $48.88(10.48)$ & $52.84(9.26)$ & .028 & -0.40 \\
Physical functioning (SF-36 PF) & $64.68(28.71)$ & $82.34(21.78)$ & $<.001$ & -0.69 \\
Role physical (SF-36 RP) & $55.24(40.52)$ & $82.66(30.23)$ & $<.001$ & -0.77 \\
Bodily pain (SF-36 BP) & $67.56(27.38)$ & $76.97(20.75)$ & .033 & -0.39 \\
General health (SF-36 GH) & $51.87(26.20)$ & $71.40(18.47)$ & $<.001$ & -0.86 \\
Vitality (SF-36 VT) & $43.55(24.78)$ & $62.66(19.91)$ & $<.001$ & -0.85 \\
Social functioning (SF-36 SF) & $73.59(26.60)$ & $87.70(18.87)$ & $<.001$ & -0.61 \\
Role emotional (SF-36 RE) & $72.58(36.97)$ & $88.17(27.72)$ & .009 & -0.48 \\
Mental health (SF-36 MH) & $74.00(17.94)$ & $79.10(18.64)$ & .124 & -0.28 \\
\hline
\end{tabular}

differences on sleep (PSQI), SF-36 mental component score (MCS), or the SF-36 subscales for bodily pain (BP) or mental health (MH) ( $p$ values> $>010)$. The pattern of significant results was unchanged when including race and ethnicity as covariates.

\section{Categorical QOL outcomes}

Significantly more TKI participants than NC participants met the CES-D clinical cutoff of 16 for depression (TKI $n=23,37 \%$; NC $n=9,15 \%, p=.004)$ and the FSI cutoff of 3 for clinically meaningful fatigue interference (TKI $n=26$, $42 \%$; NC $n=6,10 \%, p<.001$ ) and fatigue severity (TKI $n=$ $42,68 \%$; NC $n=21,34 \%, p<.001)$. In contrast, there were no group differences in the number of participants who met the PSQI clinical cutoff of 5 for clinically significant sleep disturbances (TKI $n=25,40 \%$; NC $n=25,40 \%, p=1.00$ ).

\section{Symptom burden}

To determine which symptoms were more distressing to patients relative to controls, we evaluated the individual MSAS-SF items. Symptom frequencies and the average distress ratings are presented in Table 4. Significant group differences ( $p$ values $\leq .006)$ and medium to large effect sizes in distress ratings were evident for nausea, appearancerelated concerns ("I don't look like myself"), diarrhea, changes in skin, swelling of arms or legs, itching, dizziness, and cough. For each of these symptoms, patients reported higher distress ratings than controls. Controlling for race and ethnicity, the pattern of significant results remained the same, with the exception that group differences in cough were no longer significant $(p=.036)$.

\section{Discussion}

In this study, we found that CML patients taking a TKI have worse QOL in several respects compared to age- and gender-matched people with no history of cancer. Specifically, compared to the control group, CML patients taking a TKI reported more depression, anxiety, fatigue, symptom burden (including skin changes, nausea, diarrhea, edema, itching, dizziness, and changes in appearance), and worse physical QOL. The magnitude of these differences generally reflected medium to large size effects. In contrast, there were no differences between groups in mental QOL or sleep quality.

The primary aim of this study was to compare QOL outcomes in CML patients taking TKIs to the control group. The findings that CML patients taking a TKI reported worse physical QOL and that there were no group differences in mental QOL is consistent with prior work [6]. The current study adds to the literature by demonstrating that patients taking a TKI for CML have higher levels of fatigue and depression relative to age- and gender-matched controls. Furthermore, we found that TKI patients are more likely to meet established cutoffs for clinically meaningful levels of fatigue and depression.

The second aim was to identify differences in symptom reports between the two groups. Compared to controls, patients taking a TKI reported significantly more distress related to changes in skin, itching, nausea, diarrhea, dizziness, swelling of the arms or legs, and appearance. These results are consistent with prior findings that patients taking TKIs may experience rashes, gastrointestinal upset, and edema $[1,6,21,22]$. The current finding of group differences in dizziness is somewhat surprising because although patients in some studies of imatinib have reported dizziness 
Table 4 Symptoms reported and unadjusted mean distress ratings. $N=124$

\begin{tabular}{|c|c|c|c|c|c|c|}
\hline Symptom & TKI \% & $\mathrm{NC} \%$ & TKI distress, mean (SD) & $\mathrm{NC}$ distress, mean (SD) & $\mathrm{p}^{\mathrm{c}}$ & Cohen's $d^{\mathrm{c}}$ \\
\hline Feeling drowsy & 63 & 44 & $1.38(1.25)$ & $0.85(1.04)$ & .012 & 0.46 \\
\hline Pain & 56 & 52 & $1.46(1.43)$ & $1.14(1.23)$ & .180 & 0.24 \\
\hline Difficulty concentrating & 50 & 32 & $1.12(1.22)$ & $0.63(0.98)$ & .015 & 0.44 \\
\hline Nausea & 45 & 6 & $0.98(1.23)$ & $0.12(0.45)$ & $<.001$ & 0.93 \\
\hline Itching & 40 & 16 & $0.90(1.27)$ & $0.26(0.61)$ & $<.001$ & 0.64 \\
\hline Cough & 39 & 18 & $0.75(1.04)$ & $0.30(0.73)$ & .006 & 0.50 \\
\hline Changes in skin & 35 & 3 & $0.74(1.10)$ & $0.10(0.59)$ & $<.001$ & 0.73 \\
\hline Dry mouth & 34 & 19 & $0.74(1.17)$ & $0.32(0.76)$ & .021 & 0.43 \\
\hline Feeling bloated & 34 & 23 & $0.86(1.31)$ & $0.45(0.90)$ & .043 & 0.36 \\
\hline Shortness of breath ${ }^{\mathrm{a}}$ & 34 & 18 & $0.85(1.27)$ & $0.37(0.87)$ & .016 & 0.44 \\
\hline Sweats & 34 & 19 & $0.75(1.14)$ & $0.41(0.89)$ & .070 & 0.33 \\
\hline Numbness/tingling in hands or feet & 32 & 23 & $0.77(1.21)$ & $0.46(0.92)$ & .112 & 0.29 \\
\hline Diarrhea $^{a}$ & 31 & 6 & $0.68(1.11)$ & $0.06(0.26)$ & $<.001$ & 0.77 \\
\hline Dizziness & 31 & 10 & $0.65(1.05)$ & $0.17(0.54)$ & .002 & 0.57 \\
\hline Lack of appetite & 31 & 13 & $0.72(1.23)$ & $0.26(0.72)$ & .011 & 0.46 \\
\hline Problems with sexual interest or activity ${ }^{\mathrm{b}}$ & 31 & 23 & $0.97(1.55)$ & $0.55(1.09)$ & .086 & 0.31 \\
\hline Constipation & 29 & 13 & $0.67(1.15)$ & $0.31(0.87)$ & .051 & 0.35 \\
\hline "I don't look like myself" & 29 & 2 & $0.79(1.33)$ & $0.03(0.20)$ & $<.001$ & 0.80 \\
\hline Swelling of arms or legs & 26 & 2 & $0.62(1.15)$ & $0.04(0.30)$ & $<.001$ & 0.69 \\
\hline Problems with urination & 21 & 15 & $0.50(1.07)$ & $0.37(0.99)$ & .487 & 0.13 \\
\hline Change in the way food tastes & 18 & 3 & $0.48(1.11)$ & $0.09(0.50)$ & .014 & 0.45 \\
\hline Hair loss & 15 & 8 & $0.35(0.93)$ & $0.14(0.49)$ & .126 & 0.28 \\
\hline Weight loss & 11 & 5 & $0.22(0.65)$ & $0.04(0.17)$ & .037 & 0.38 \\
\hline Mouth sores & 8 & 3 & $0.15(0.58)$ & $0.05(0.29)$ & .209 & 0.22 \\
\hline Vomiting & 8 & 0 & $0.14(0.51)$ & $0(0)$ & .031 & 0.39 \\
\hline Difficulty swallowing & 5 & 2 & $0.13(0.58)$ & $0.03(0.20)$ & .190 & 0.23 \\
\hline
\end{tabular}

TKI group patients treated with tyrosine kinase inhibitors, NC group noncancer participants, MSAS-SF Memorial Symptom Assessment ScaleShort Form

${ }^{\text {a }}$ Data missing for one participant

${ }^{\mathrm{b}}$ Data missing for two participants

${ }^{\mathrm{c}} P$ values and Cohen's $d$ compare distress ratings for TKI and NC participants

$[1,21]$, it is less common than other symptoms, such as muscle cramps $[1,6,21]$. Nonsignificant findings for pain were also surprising. Although musculoskeletal pain has been noted to be a common symptom in patients on imatinib $[1,6]$ we detected no group differences in pain. Of note, pain was the most distressing symptom reported by noncancer participants and more than half of the participants in both groups reported experiencing pain in the past week. Another unexpected finding was group differences in distress over appearance with nearly one-third of the patients endorsing this item. Although there were no group differences in distress over hair loss, alopecia is a possible side effect of imatinib and nilotinib [22]. To our knowledge, other studies have not assessed patients' concerns about their appearance. Nonetheless, we found that changes in appearance, whether due to skin changes, edema, or other factors, are distressing for patients taking TKIs. Finally, in the current study, differences in coughing between TKI and control participants were no longer significant at the $p<.01$ level when controlling for race and ethnicity. More research is needed in a larger, more diverse sample to understand race- and ethnicityrelated differences in patient-reported symptoms.

A strength of this study is the inclusion of an age- and gender-matched comparison group of people without cancer. Limitations include the small sample size and the use of a cross-sectional study design. It is also possible that patients who participated had better QOL and less symptomatology than patients who elected not to participate. Another limitation is the inclusion of patients prescribed three different treatments for CML. Although including three different TKI agents increases the generalizability of results, the small number of patients taking any one agent 
precludes meaningful statistical comparisons among these treatment subgroups. The three agents may differentially affect QOL. For example, pleural effusions (of which dry cough, chest pain, and dyspnea could be symptoms) may be more common among patients taking dasatinib [23], rashes may be more common in those taking nilotinib [22], and gastrointestinal upset and edema may be more common among patients taking imatinib [22]. Finally, because our control participants did not have CML, it is not possible to separate effects of treatment from effects of disease.

In summary, this study provides strong evidence that CML patients taking imatinib, nilotinib, or dasatinib experience more depression, more fatigue, greater symptom burden, and worse physical QOL compared to people with no history of cancer matched on age and gender. Furthermore, patients taking TKIs are more likely to report clinically meaningful depression and fatigue. These findings point to the need to develop interventions that can address QOL issues in CML patients taking TKIs.

Acknowledgments The authors would like to thank the following individuals for their contribution to this work: Charissa Hicks, BA; Darcie Deaver, ARNP; and Patricia Cabrera, BA. The authors are grateful to the Moffitt Survey Methods Core for assistance with data management.

Conflict of interest JP-I has received research funding and honoraria and provided consultancy for Novartis and Bristol-Myers Squibb.

\section{References}

1. O'Brien SG, Guilhot F, Larson RA et al (2003) Imatinib compared with interferon and low-dose cytarabine for newly diagnosed chronic-phase chronic myeloid leukemia. N Engl J Med 348 (11):994-1004

2. Hahn EA, Glendenning GA, Sorensen MV et al (2003) Quality of life in patients with newly diagnosed chronic phase chronic myeloid leukemia on imatinib versus interferon alfa plus low-dose cytarabine: results from the IRIS Study. J Clin Oncol 21 (11):2138-2146

3. Aziz Z, Iqbal J, Aaqib M et al (2011) Assessment of quality of life with imatinib mesylate as first-line treatment in chronic phasechronic myeloid leukemia. Leuk Lymphoma 52(6):1017-1023

4. Agrawal M, Garg RJ, Cortes J et al (2010) Tyrosine kinase inhibitors: the first decade. Curr Hematol Malig Rep 5(2):70-80

5. Pinilla-Ibarz J, Cortes J, Mauro MJ (2011) Intolerance to tyrosine kinase inhibitors in chronic myeloid leukemia: definitions and clinical implications. Cancer 117(4):688-697
6. Efficace F, Baccarani M, Breccia M et al (2011) Health-related quality of life in chronic myeloid leukemia patients receiving longterm therapy with imatinib compared with the general population. Blood 118(17):4554-4560

7. Apolone G, Mosconi P (1998) The Italian SF-36 Health Survey: translation, validation and norming. J Clin Epidemiol 51 (11):1025-1036

8. Jacobsen PB, Donovan KA, Small BJ et al (2007) Fatigue after treatment for early stage breast cancer: a controlled comparison. Cancer 110(8):1851-1859

9. Hann DM, Jacobsen PB, Azzarello LM et al (1998) Measurement of fatigue in cancer patients: development and validation of the Fatigue Symptom Inventory. Qual Life Res 7(4):301-310

10. Broeckel JA, Jacobsen PB, Horton J et al (1998) Characteristics and correlates of fatigue after adjuvant chemotherapy for breast cancer. J Clin Oncol 16(5):1689-1696

11. Donovan KA, Jacobsen PB, Small BJ et al (2008) Identifying clinically meaningful fatigue with the Fatigue Symptom Inventory. J Pain Symptom Manage 36(5):480-487

12. Radloff LS (1977) The CES-D scale: a self-report depression scale for research in the general population. Appl Psychol Meas 1:385401

13. Hann D, Winter K, Jacobsen P (1999) Measurement of depressive symptoms in cancer patients: evaluation of the Center for Epidemiological Studies Depression Scale (CES-D). J Psychosom Res 46(5):437-443

14. Buysse DJ, Reynolds CF 3rd, Monk TH et al (1989) The Pittsburgh Sleep Quality Index: a new instrument for psychiatric practice and research. Psychiatry Res 28(2):193-213

15. Carpenter JS, Andrykowski MA (1998) Psychometric evaluation of the Pittsburgh Sleep Quality Index. J Psychosom Res 45(1):513

16. Beck SL, Schwartz AL, Towsley G et al (2004) Psychometric evaluation of the Pittsburgh Sleep Quality Index in cancer patients. J Pain Symptom Manage 27(2):140-148

17. Chang VT, Hwang SS, Feuerman M et al (2000) The memorial symptom assessment scale short form (MSAS-SF). Cancer 89 (5):1162-1171

18. Ware J (1993) SF-36 health survey: Manual and interpretation guide. The Health Institute, New England Medical Center, Boston

19. Ware JE, Kosinski M, Keller SD (1994) SF-36 pysical and mental health summary scales: A user's manual. The Health Institute, New England Medical Center, Boston

20. Cohen J (1988) Statistical power analysis for the behavioral sciences. Lawrence Erlbaum Associates

21. Gambacorti-Passerini C, Antolini L, Mahon FX et al (2011) Multicenter independent assessment of outcomes in chronic myeloid leukemia patients treated with imatinib. J Natl Cancer Inst 103 (7):553-561

22. Saglio G, Kim DW, Issaragrisil S et al (2010) Nilotinib versus imatinib for newly diagnosed chronic myeloid leukemia. N Engl J Med 362(24):2251-2259

23. Masiello D, Gorospe G 3rd, Yang AS (2009) The occurrence and management of fluid retention associated with TKI therapy in CML, with a focus on dasatinib. J Hematol Oncol 2:46 